\title{
Self-phase modulation effects and pulse propagation in gain-guided fibers
}

Renjie Zhou

\section{Baldemar Ibarra-Escamilla \\ Qiwen Zhan}

Peter E. Powers

\section{Joseph W. Haus}

joseph.haus@notes.udayton.edu
Electro-Optics Program, University of Dayton, 300 College Park, Dayton OH 45469, USA

Instituto Nacional de Astrofisica, Optica y Electronica, Luis Enrique Erro No. 1 Puebla, Pue 72000, México

Electro-Optics Program, University of Dayton, 300 College Park, Dayton OH 45469, USA

Electro-Optics Program, University of Dayton, 300 College Park, Dayton OH 45469, USA Physics Department, University of Dayton, 300 College Park, Dayton OH 45469, USA

Electro-Optics Program, University of Dayton, 300 College Park, Dayton OH 45469, USA

We present a theoretical analysis of pulse propagation and self-focusing in a gain-guided (GC) fiber amplifier. A weak pulse is launched in the GG fiber when the input pulse reaches a critical power the pulse begins to collapse in the transverse direction. By using different input powers the transmission characteristics are changed. We add coupling to a single-mode fiber at the output end and study pulse dispersion and energy. [DOI: 10.2971/jeos.2009.09051]

Keywords: fiber laser, fiber nonlinear optics, transverse effects in nonlinear optics, self-action effects

\section{INTRODUCTION}

New fiber designs have enabled an increase in power extracted from fiber amplifiers and lasers. In particular Large Mode Area (LMA) fibers suppress nonlinear effects in fibers, thus allowing higher powers before nonlinear optical effects, such as Stimulated Brillouin Scattering (SBS) or Stimulated Raman Scattering (SRS), are an issue [1, 2]. For many applications, it is desired that the output signal is a single longitudinal mode, but that requires effective means to suppress other cavity modes. For instance, the use of a multicore fiber has been recently proposed and experimentally examined to produce a large area single mode fiber [3]. Exploiting indexmatched core-cladding fiber designs improved higher-order mode suppression can be achieved in solid or microstructured fibers [4]. This is a first step to help to narrow the optical spectrum and sustain high power. Finite-difference simulations show that even gentle bends needed for a coiled-fiber configuration can lead to unintended resonant coupling and excessive loss in the fundamental mode, ruining the selective mode suppression.

Optical fibers are generally made with guiding in the core using total internal reflection to create the guided mode. However, Siegman has proposed a scheme for gain guiding in optical fibers [5, 6] and experimenters [7] have recently shown that one can use an index, anti-guiding core with a highly doped amplifying large region to create a stable, single mode output by gain guiding the light in the fiber. The large mode area of these fibers with gain in the core is reminiscent of unstable cavity designs for high power lasers.
In this paper, we simulate propagation of spatio-temporal pulses in gain-guided (GG) fibers. We examine the effect of third-order nonlinearity, self-phase modulation on pulse propagation in GG fibers. In a two-dimensional geometry the third-order nonlinearity leads to the self-focusing and collapse of the beam into a filament, unless the collapse is arrested by other processes. At the same time, the nonlinear index induced spatial dispersion leads to an additional temporal dispersion for the pulse. The numerical modeling of propagation is performed with different GG fiber parameters and the detailed results are examined.

\section{NUMERICAL SIMULATIONS}

In a standard step-index fiber with core radius $a$ and core and cladding indices $n_{c o}$ and $n_{c l},\left(\Delta n=n_{c o}-n_{c l}\right)$, the number of guided modes and the mode profile are determined by the $V$ parameter, whose square in a low index contrast limit is given by:

$$
V^{2}=\Delta N=2 n_{0}\left(\frac{2 \pi a}{\lambda}\right)^{2} \Delta n,
$$

where $n_{0}$ is the average index of the core-cladding materials, and $\lambda$ is the signal wavelength. However, the $V$ parameter for a GG fiber is defined by Siegman as a complex parameter $[5,6]$

$$
\widetilde{V}^{2}=\Delta N+i G
$$

The real part is the usual $V$ parameter, which is defined in 
Eq. (1), and the imaginary part is the gain parameter which is given by:

$$
G=\frac{n_{0} \lambda}{2 \pi}\left(\frac{2 \pi a}{\lambda}\right)^{2} g
$$

where $g$ is the power gain coefficient in the core. For $\Delta n>0$ and $g=0$, the fiber corresponds to the usual guided fiber geometry. For gain guided fibers, the solutions of propagating modes can be written in terms of Bessel functions with complex parameters which are related to index contrast and the gain in the waveguide as given in Eqs. (1) and (2) [5, 6].

The GG fibers could have large core radii which could provided high power output while lasing still maintains a single mode characteristic. Recent experiments have demonstrated single mode and stable lasing in a gain-guided, index antiguided $\mathrm{Nd}^{3+}$ doping fiber with a highly doped amplifying large core region. The fiber was initially pumped with a flashlamp, and recently laser diode pump was also realized [7, 8].

The pulse propagation in the GG fiber is governed by the Nonlinear Schrödinger Equation (NLSE). We assume a slowing varying amplitude $A(x, y, z, \tau)$, so the NLSE under paraxial approximation is written in the form below.

$$
\begin{aligned}
\frac{\partial A}{\partial z}=\frac{i}{2 k} \nabla_{\perp}^{2} A+\frac{i}{2 k} & \left(\frac{\omega}{c}\right)^{2}\left(n_{c o}^{2}-n_{c l}^{2}\right) A \\
& -i \frac{\beta_{2}}{2} \frac{\partial^{2} A}{\partial \tau^{2}}+i \gamma|A|^{2} A-\frac{\alpha}{2} A,
\end{aligned}
$$

where $\beta_{2}$ is the group velocity dispersion parameter, which is small for our numerical parameters; in silica glass it is $-26 \mathrm{fs}^{2} / \mathrm{mm}$ and in single mode fiber it is $-20 \mathrm{fs}^{2} / \mathrm{mm}$ [9]. For 1 ps pulse widths and $10 \mathrm{~cm}$ GG fiber length the temporal dispersion is negligible although we retained it in our simulations. $k$ is the wave number in the cladding of the fiber given by $(\omega / c) n_{c l}$, where $\omega$ is the central frequency of the pulse, $c$ is the velocity of light, $n_{c l}$ is the cladding refractive index which is 1.5689 in the GG fiber, as taken from [7], and $n_{c o}$ is a complex core index. The results are not sensitive to the real part of the core and cladding indices. The fiber can be index guiding or antiguiding with no noticeable change of the mode profile. The complex core index consists of a refractive index and the gain modified index, and it is written as $n_{c o}=n_{r}+i n_{i}$, where $n_{r}=1.5734$ is the real part of the index [7], and $n_{i}$ is the imaginary part, and $n_{i}=-g / 2 k_{0}$, where $g=7 \times 10^{-5} \mu \mathrm{m}^{-1}$ $\left(0.7 \mathrm{~cm}^{-1}\right)$ is the power gain coefficient. $n_{c l}$ is the cladding index, $k_{0}=2 \pi / \lambda_{\text {vac }}$, is the wave number in vacuum and $\lambda_{\text {vac }}$ is the central wavelength of the pulse in vacuum, which is $1.55 \mu \mathrm{m}$. The nonlinearity coefficient $\gamma=k_{0} n_{2}$, where $n_{2}$ is the nonlinear index in silica, which is $3.0 \times 10^{-8} \mu \mathrm{m}^{2} / \mathrm{W}[9,10]$, and $\alpha$ is an absorption coefficient, which is assumed to be negligible in our simulations.

Cylindrical symmetry is assumed, i.e. $A(r, z, \tau)$, and we apply the 1-D Hankel transform, instead of a 2-D Fourier transform, to the transverse direction, making the numerical simulations run much faster and saving computational memory. In the time domain, we apply 1-D Fourier transform. For numerical simulations, Eq. (4) is written in terms of operators:

$$
\frac{\partial A}{\partial z}=\mathcal{L}_{r} A+\mathcal{L}_{D} A+\mathcal{L}_{I} A+\mathcal{N}(A) \cdot A,
$$

where

$$
\mathcal{L}_{r}=\left(\frac{i}{2 k}\right) \nabla_{\perp}^{2}
$$

is the diffraction operator,

$$
\mathcal{L}_{D}=-i\left(\frac{\beta_{2}}{2}\right) \frac{\partial^{2}}{\partial \tau^{2}}
$$

is the dispersion term,

$$
\mathcal{L}_{I}=\left(\frac{i}{2 k}\right)\left(\frac{\omega}{c}\right)^{2}\left(n_{c o}^{2}-n_{c l}^{2}\right)
$$

is the complex index term, which includes gain guiding in the core, and $\mathcal{N}(A)=i \gamma|A|^{2}$ is the nonlinear self-phase modulation term. The solution of Eq. (5) for a step size $\Delta z$ is written in the form below:

$$
A(z+\Delta z)=Z\left\{\exp \left[\int_{0}^{\Delta z}\left(\mathcal{L}_{r}+\mathcal{L}_{D}+\mathcal{N}(A)+\mathcal{L}_{I}\right) d z\right]\right\}
$$

where $Z\{\ldots\}$ is the normal order of operators with respect to z. Eq. (9) can be approximately written as:

$$
\begin{aligned}
A(z+\Delta z) & =e^{\left(\mathcal{L}_{r}+\mathcal{L}_{D}\right) \frac{\Delta z}{2}} \cdot e^{\mathcal{L}_{I} \Delta z} \cdot e^{\mathcal{N}(A) \Delta z} \cdot e^{\left(\mathcal{L}_{r}+\mathcal{L}_{D}\right) \frac{\Delta z}{2}} A(z) \\
& =e^{\mathcal{L}_{r} \frac{\Delta z}{2}} \cdot e^{\mathcal{L}_{D} \frac{\Delta z}{2}} \cdot e^{\mathcal{L}_{I} \Delta z} \cdot e^{\mathcal{N}(A) \Delta z} \cdot e^{\mathcal{L}_{r} \frac{\Delta z}{2}} \cdot e^{\mathcal{L}_{D} \frac{\Delta z}{2}} A(z) .
\end{aligned}
$$

Eq. (10) is the split-step method for solving the propagation. The action of each operator is described below. The pulse propagation under temporal dispersion can be described as:

$$
A\left(z+\frac{\Delta z}{2}\right)=e^{\mathcal{L}_{D} \frac{\Delta z}{2}} A(z) .
$$

Eq. (11) can be solved by using a 1-D Fast Fourier Transform (FFT) in time domain, and the field at $z+\Delta z / 2$ is:

$$
A\left(z+\frac{\Delta z}{2}\right)=F F T^{-1}\left\{\exp \left[-i \frac{\beta_{2} \omega^{2}}{4} \Delta z\right] F F T\{A(z)\}\right\}
$$

where $\omega$ denotes the temporal angular frequency in the Fourier space. For the diffractive propagation, we apply the Fast Hankel Transform (FHT). The 2-D Laplacian operator of the diffraction is written in cylindrical coordinate as:

$$
\nabla_{\perp}^{2}=\frac{\partial^{2}}{\partial r^{2}}+\frac{1}{r} \frac{\partial}{\partial r}
$$

where $r^{2}=x^{2}+y^{2}$. By using the FHT, then we have:

$$
\frac{i}{2 k}\left(\frac{\partial^{2}}{\partial r^{2}}+\frac{1}{r} \frac{\partial}{\partial r}\right) A(r, t) \stackrel{F H T}{\longrightarrow}-\frac{i}{2 k} 4 \pi^{2} \rho^{2} A_{\mathcal{H}}(\rho, t),
$$

where $A_{\mathcal{H}}(\rho, t)$ is the Hankel transform of $A(r, t)$ and $\rho$ is the spatial radial frequency, and the transform is defined as:

$$
A_{\mathcal{H}}(\rho, t)=2 \pi \int_{0}^{\infty} A(r, t) J_{0}(\rho \cdot r) d r
$$

where $J_{0}(\ldots)$ denotes the zero-order of Bessel function of the first kind. Also, note that the forward and inverse Hankel transform take the same form as Eq. (15). A quasi-discrete 

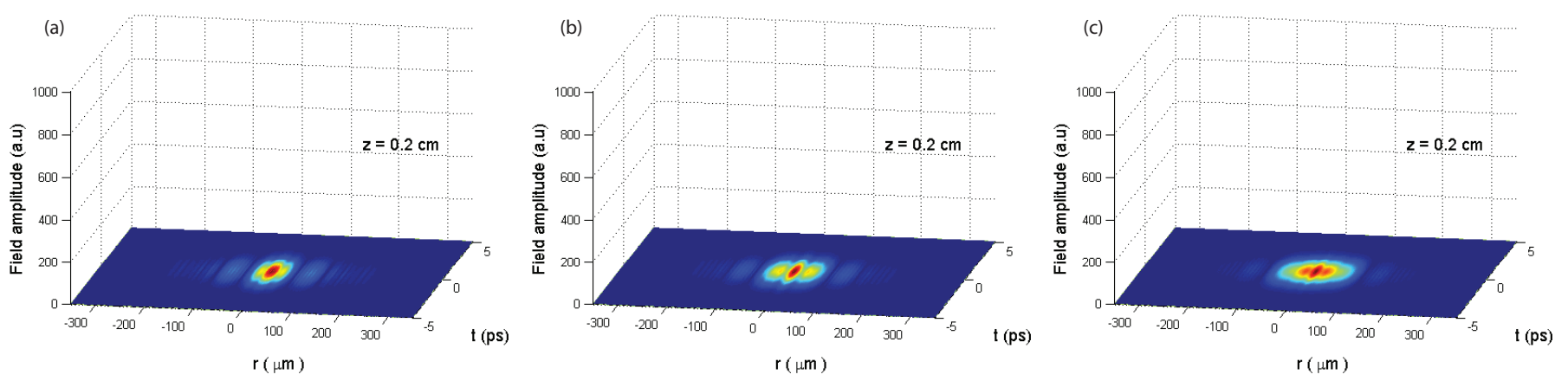

FIG. 1 The spatio-temporal pulses propagation in the GG fiber. (a)-(c) are pulses propagation for $50 \mu \mathrm{m}, 75 \mu \mathrm{m}$, and $100 \mu \mathrm{m}$ core size respectively (size: $700 \mathrm{kB}$, format: avi, see Fig1a.avi, Figıb.avi, Fig1c.avi).

Hankel transform is used to evaluate the zero-order transform $[11,12]$. The field after propagation of $\Delta z / 2$ is [13]:

$$
A\left(z+\frac{\Delta z}{2}\right)=F H T\left\{\exp \left[-i \frac{\pi^{2}}{k} \rho^{2} \Delta z\right] A_{\mathcal{H}}(\rho, t)\right\} .
$$

There are many other numerical methods for solving the NLSE. For example, the finite difference method, such as the Crank-Nicolson algorithm, with transparent boundary condition can be applied to solve the pulse propagation [14, 15]. We have also applied the 2D FFT method assuming no cylindrical symmetry to the propagation equations without temporal effects and found identical results as a check of the FHT algorithm.

The complex index parameter includes gain-guiding effects, the field for the index term is:

$$
A(z+\Delta z)=\exp \left[\frac{i}{2 k}\left(\frac{\omega}{c}\right)^{2}\left(n_{c o}^{2}-n_{c l}^{2}\right) \Delta z\right] \cdot A(z)
$$

and for nonlinear terms, the field at $z+\Delta z$ is be obtained through:

$$
A(z+\Delta z)=\exp \left(i \gamma \cdot|A(z)|^{2} \cdot \Delta z\right) \cdot A(z)
$$

After solving for each material process, the pulse propagates under the action of dispersion and diffraction for another $\Delta z / 2$ step, as depicted in Eq. (10), and the pulse at $z+\Delta z$ is used for the next step. Without self-phase modulation the mode profile takes the form given by Siegman [6],

$$
\tilde{E}_{01}(r)=\left\{\begin{array}{ll}
J_{0}(\tilde{u} r / a) & r \leq a \\
K_{0}(\tilde{w} r / a)\left[J_{0}(\tilde{u}) / K_{0}(\tilde{w})\right] & r \geq a
\end{array},\right.
$$

where $\tilde{w}=w_{r}+i w_{i}, w_{r} \cong-\left(j_{01}^{2} / \Delta N\right) \times\left[G / G_{t h}-1\right]$ and $w_{i} \cong \sqrt{-\Delta N}$, where $\Delta N$ is given in Eq. (1), and $G_{t h} \cong$ $\sqrt{-133.8 / \Delta N}, j_{01} \cong 2.405$ and $\tilde{u} \cong j_{01}(\tilde{w}-1) / \tilde{w}$. With a complete propagation algorithm, the self-focusing effects from the self-phase modulation term in the NLSE are investigated in the next section.

\section{RESULTS}

Gain-guided fibers are studied with three different core radii which are $50 \mu \mathrm{m}, 75 \mu \mathrm{m}$, and $100 \mu \mathrm{m}$ respectively. For each case, we simulated the self-focusing and the self-phase modulation effect in the GG fiber. At the end of the GG fiber the pulses are coupled into single mode fiber which has a core radius of $4.5 \mu \mathrm{m}$. The transmission and the phase characteristics of the pulse are looked at in the single mode fiber. The input pulse to the GG fiber is defined as Gaussian function in space and time:

$$
A(r, t)=A_{0} \exp \left[-\left(1+j \cdot P_{\text {curve }}\right) \cdot \frac{r^{2}}{2 w_{0}^{2}}\right] \cdot \exp \left[-\frac{t^{2}}{2 \tau^{2}}\right],
$$

where $A_{0}$ is the initial field amplitude of $2.0 \mathrm{~W}^{1 / 2} \cdot \mu \mathrm{m}^{-1}, w_{0}$ is the transverse pulse width which is taken as $50 \mu \mathrm{m}$, and $\tau=1 \mathrm{ps}$ is the pulse width, and the initial temporal phase curvature is $P_{\text {curve }}=0$, which means the initial pulse is not chirped. The pulses with large enough initial field amplitude will self-focus immediately before collapsing, the simulations stop at that point since the beam collapse introduces high spatial frequencies that are not captured by our numerical simulations.

\subsection{Pulse propagation in GG fiber with self-focusing, and beam collapse}

The spatio-temporal pulse propagation in the GG fiber is simulated, and the evolution of the field amplitude are shown by the animations in Figures 1(a)-(c) with $50 \mu \mathrm{m}, 75 \mu \mathrm{m}$, and $100 \mu \mathrm{m}$ core radii respectively. Initially, the pulses oscillate in the transverse direction. This is because the pulses are coupled not only into the fundamental mode, but also to the higher order modes in the fiber. However, after the pulses obtain sufficient power, they have the shape of the fundamental mode for the GG fiber, as described by Siegman. As a pulse with sufficient launched energy propagates, it starts to focus, and finally collapses into an on-axis filament. The difference between the chosen core radii is the position where the pulse collapse occurs for a given input energy. The pulses start collapsing after propagating around $11.2 \mathrm{~cm}, 9.2 \mathrm{~cm}$, and $9.0 \mathrm{~cm}$ for $50 \mu \mathrm{m}, 75 \mu \mathrm{m}$, and $100 \mu \mathrm{m}$ core radii, respectively. For smaller core radius, the distance is larger. The reason for this trend is that the pulse has smaller gain in the transverse direction in smaller cores compared with larger ones. The pulses need a critical peak power to collapse. Thus, in order to obtain the same power, the propagation distance in the smaller core size fiber should be longer. 

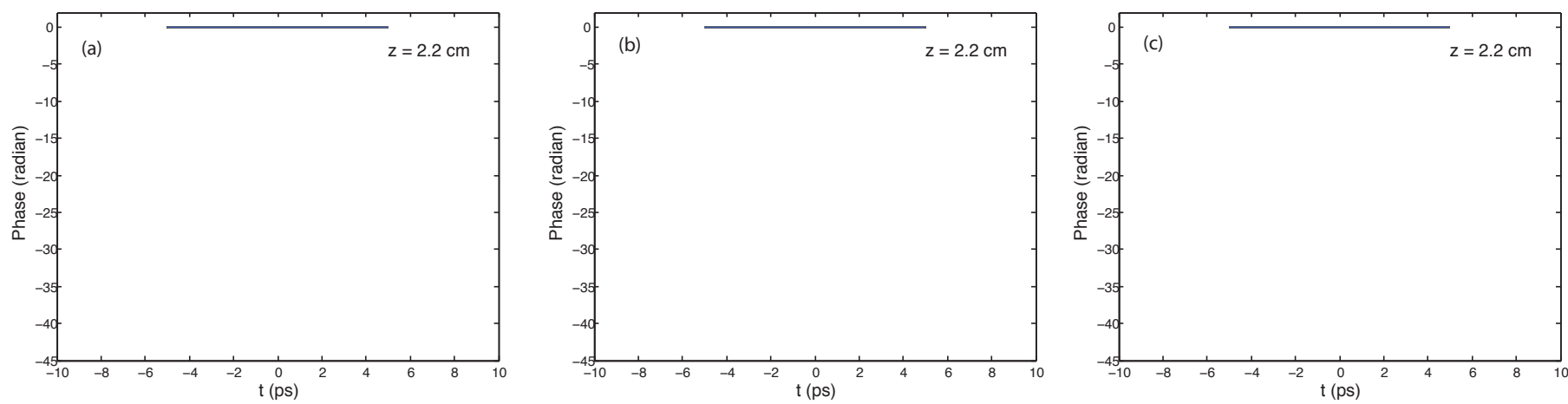

FIG. 2 On axis self-phase modulation in GG fiber. (a)-(c) are the self-phase modulations for $50 \mu \mathrm{m}$, $75 \mu \mathrm{m}$, and $100 \mu \mathrm{m}$ core size respectively (size: $400 \mathrm{kB}$, format: avi, see Figza.avi, Fig2b.avi, Fig2c.avi).
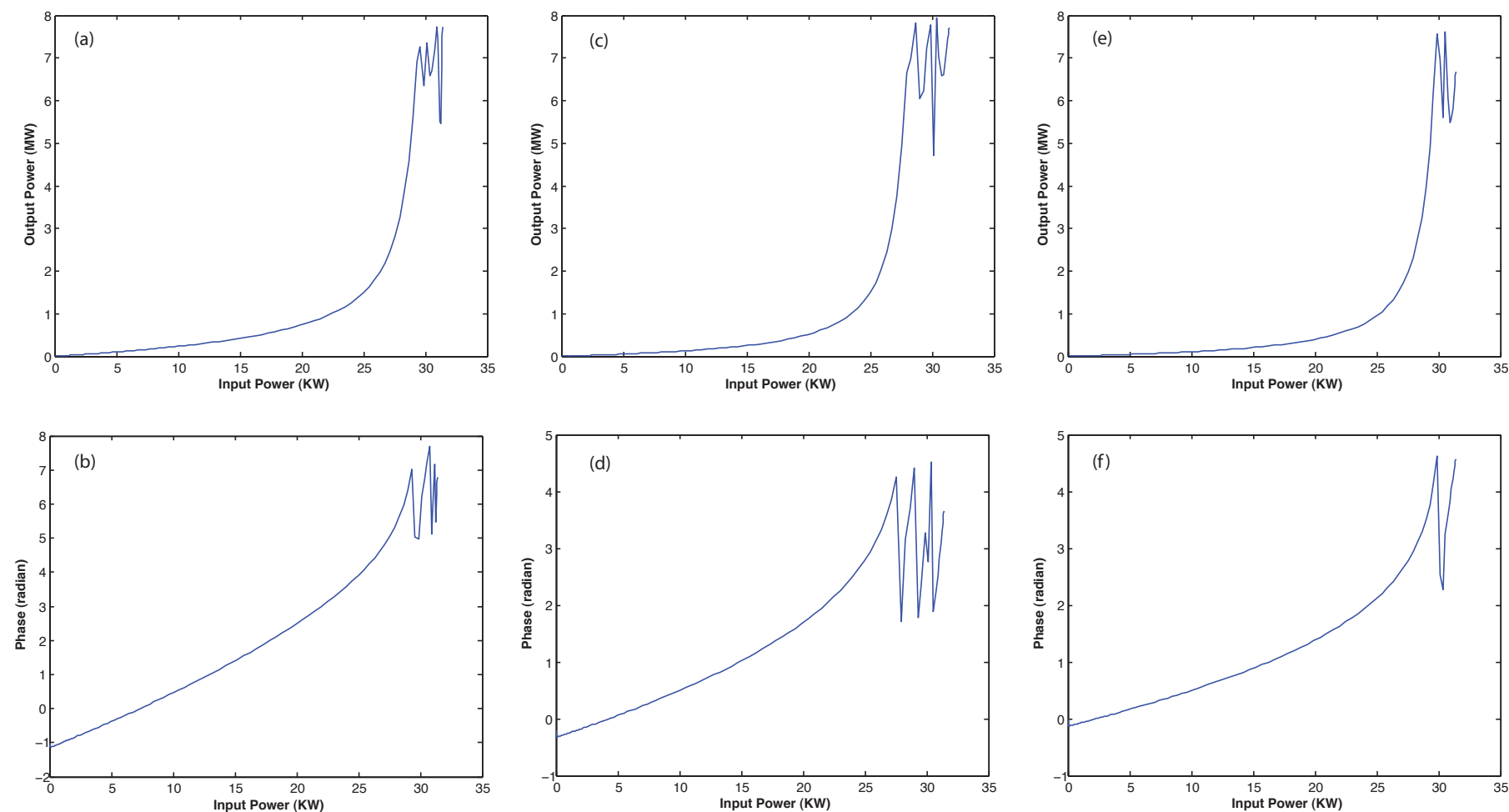

FIG. 3 Pulse coupling into the single mode fiber. (a), (c), and (e) are the transmission curves for $50 \mu \mathrm{m}, 75 \mu \mathrm{m}$, and $100 \mu \mathrm{m}$ core size respectively, and the GG fiber lengths are $11.2 \mathrm{~cm}, 9.2 \mathrm{~cm}$, and $9.0 \mathrm{~cm}$ accordingly. (b), (d), and (f) are the phase curves for $50 \mu \mathrm{m}, 75 \mu \mathrm{m}$, and $100 \mu \mathrm{m}$ core size respectively. The critical power for collapse agrees with analytical results and input powers are useful only until the beam collapses.

\subsection{Self-phase modulation effect in the GG fiber}

In this sub section, we study the on-axis phase $(r=0)$ of the pulse propagating in the GG fiber. Figures $2(\mathrm{a})-(\mathrm{c})$ show the on-axis phase evolution as the pulses propagating in the GG fiber. As the pulses propagate through the GG fiber, the phase is constant for the initial propagation distances, but the pulse develops a time varying phase at the position where the pulse starts to focus on axis, see Figure 1.

Before the pulse collapses, there is a large temporal phase change across the pulse. The critical power for pulse collapse on the axis is given by $P_{c r}=N_{c r} \lambda^{2} / 4 \pi n_{r} n_{2}$ [16] and the numerical parameter is $N_{c r} \approx 1.9$, which is about 7.8 MW for the parameters used in our simulations.

\subsection{Pulse coupling into the single mode fiber}

We couple the output pulses from the GG fiber into a single mode fiber with a Gaussian profile whose mode width is $w=4.95 \mu \mathrm{m}$ [9]. The characteristics of the transmission amplitude and phase are investigated. The results are shown in Figures 3(a)-(f). Figures 3(a), (c), (e), are the transmitted power for the single mode fiber as a function of the input to the GG fiber. The transmitted power is obtained by integrating the field intensities over the transverse direction at each time point. At lower input powers, the slope efficiency is small, which indicates the coupling efficiency from GG fiber to SM fiber is small. This is because, at smaller input power, the GG fiber mode beam width is large in transverse domain. As the input power increase, the pulse will start to focus at the end of the GG fiber. So the coupling efficiency is higher because it 
has better overlap with the single-mode fiber mode. This action results in a much higher slope efficiency. However, when the input power is too high and beam collapse occurs, the coupling efficiency precipitously drops, because the beam radius no longer matches the numerical aperture of the SM fiber. The coupling efficiency will become unstable after the point of collapse and results are not followed beyond this point. Figures 3(b), (d), (f) are the phases in the single mode fiber versus the input power. The phase curves show characteristics that are similar to the transmission curves. We note that the phase variation is bigger for the $50 \mu \mathrm{m}$ GG fiber core radius than for the other core radii.

These results can now be used to infer the spread of the pulse in the single mode fiber. A chirp of about $12 \pi$ radians across a 1 ps wide pulse corresponds to a chirp parameter of around $6 \pi$. The fiber length for dispersion compensation for shorter pulses is reduced and the compensation fiber could be inserted in a fiber laser cavity to balance the dispersion in each round trip.

\section{CONCLUSIONS}

We have studied the self-phase modulation effect on the transverse spatial collapse of a pulse in a GG fiber. The spatial collapse of the beam has an effect on the temporal dispersion and we quantify this effect in our simulations. Following the GG fiber section, the pulse was coupled into a single mode fiber to study the transmission power and temporal phase variation with launch power into the GG fiber. The saturable absorber action of the transmission can be exploited to create modelocking in a fiber cavity, i.e. Kerr lens mode-locking in fiber lasers for generating short pulses. The temporal dispersion length for our case is much longer than the GG fiber length. By shortening the pulse width to tens of femtoseconds the material dispersion length will be comparable or smaller than the GG fiber length. This situation will be explored in the context of a mode-locked fiber laser with a GG fiber section in the cavity.

\section{ACKNOWLEDGEMENTS}

RZ was supported by the University of Dayton Graduate School through a Graduate Student Summer Fellowship. BIE was supported by CONACyT grant 104551 . We are grateful to Georges Nehmetallah for discussions on fast Hankel transform algorithms.

\section{References}

[1] 0. Schmidt, J. Rothhardt, F. Röser, S. Linke, T. Schreiber, K. Rademaker, J. Limpert, S. Ermeneux, P. Yvernault, F. Salin, and A. Tünnermann, "Millijoule pulse energy Q-switched short-length fiber laser" Opt. Lett. 32, 1551-1553 (2007).

[2] J. Limpert, A. Liem, M. Reich, T. Schreiber, S. Nolte, H. Zellmer, A. Tünnermann, J. Broeng, A. Petersson, and C. Jakobsen, "Lownonlinearity single-transverse-mode ytterbium-doped photonic crystal fiber amplifier" Opt. Express 12, 1313-1319 (2004).

[3] M. M. Vogel, M. Abdou-Ahmed, A. Voss, and T. Graf, "Very-largemode-area, single-mode multicore fiber" Opt. Lett. 34, 2876-2878 (2009).

[4] J. Fini, "Design of solid and microstructure fibers for suppression of higher-order modes" Opt. Express 13, 3477-3490 (2005).

[5] A. E. Siegman, "Propagating modes in gain-guided optical fibers" J. Opt. Soc. Am. A 20, 1617-1628 (2003).

[6] A. E. Siegman, "Gain-guided, index-anti-guided fiber lasers" J. Opt. Soc. Am. B 24, 1677-1682 (2007).

[7] Y. Chen, V. Sudesh, T. McComb, M. C. Richardson, M. Bass, and J. Ballato, "Lasing in a gain-guided index anti-guided fiber" J. Opt. Soc. Am. B 24, 1683-1688 (2007).

[8] V. Sudesh, T. Mccomb, Y. Chen, M. Bass, M. Richardson, J. Ballato, and A. E. Siegman, "Diode-pumped $200 \mu \mathrm{m}$ diameter core, gainguided, index-antiguided single mode fiber laser" Appl. Phys. B 90, 369-372 (2008).

[9] G. P. Agrawal, Fiber-Optic Communication Systems, (John Wiley at Sons, Inc., New York, 2002).

[10] G. P. Agrawal, Nonlinear fiber Optics, (Academic, New York, 2001).

[11] L. Yu, M. Huang, and M. Chen, "Quasi-discrete Hankel transform" Opt. Lett. 23, 409-411 (1998).

[12] M. Guizar-Sicairos, and J. Gutiérrez-Vega, "Computation of quasidiscrete Hankel transform of integer order for propagating optical wave fields" J. Opt. Soc. Am. A 21, 53-58 (2004).

[13] G. Nehmetallah, and P. P. Banerjee, "Stabilization of a (D+1)dimensional dispersion-managed solutions in Kerr media by an alternating dispersion structure" J. Opt. Soc. Am. B 23, 203-211 (2006).

[14] G. R. Hadley, "Transparent boundary conditions for beam propagation" Opt. Lett. 16, 624-626 (1991).

[15] S. T. Hendow, and S. A. Shakir, "Recursive numerical solution for nonlinear wave propagation in fibers and cylindrical symmetric system" Appl. Opt 25, 1759-1764 (1986).

[16] G. Fibich, and A. L. Gaeta, "Critical power for self-focusing in bulk media and in hollow waveguides" Opt. Lett. 25, 335-337 (2000). 